\title{
DINÁMICA DEL AZUFRE BAJO CONDICIONES DE INVERNADERO DE UN TYPIC SULFAQUEPT EN EL VALLE MEDIO DEL SINÚ, COLOMBIA
}

\section{SULPHUR DYNAMIC OF A TYPIC SULFAQUEPT UNDER GREENHOUSE CONDITIONS IN THE MID SINU RIVER VALLEY, COLOMBIA}

\author{
Eliécer M. Cabrales ${ }^{1}$, Garrnier Grandeth ${ }^{1}$ y Alvaro García ${ }^{2}$ \\ Recibido para evaluación: Septiembre 1 de 2007 - Aceptado para publicación: Agosto 7 de 2008
}

\section{RESUMEN}

La presente investigación se hizo en los tres primeros horizontes de un suelo sulfatado ácido en tres sitios ubicados en el transecto Berastegui - Mimbre, municipio de Ciénaga de Oro, Colombia, ubicada a 15 m.s.n.m., en un bosque seco tropical (Bs-t). La investigación consistió en evaluar en invernadero la dinámica del azufre en un suelo sulfatado ácido del Valle del Sinú, para lo cual se utilizó un diseño completamente al azar con arreglo factorial, con dos factores (sitio y horizonte). Dentro de cada uno de los factores se evaluaron tres niveles con cuatro repeticiones en cada una de ellas, para un total de 36 unidades experimentales. Los parámetros evaluados fueron $\mathrm{S}$ total, S orgánico, S inorgánico, S extraíble, sulfato soluble, los cuales se determinaron desde que el suelo estaba completamente saturado, hasta que quedó seco al aire. Se encontró que la dinámica de la humedad sólo influyó en el contenido de sulfatos, los cuales disminuyeron con el drenado del suelo.

Palabras claves: suelo sulfatado ácido, azufre total, azufre orgánico, azufre inorgánico, sulfatos.

\begin{abstract}
The three first horizons of a sulphate acidic soil in three different sites located between Berastegui and Mimbre (Cienaga de Oro, Cordoba, Colombia) with $15 \mathrm{~m}$ above sea level, in a tropical dry forest (Bs-t) were analyzed. The sulphur dynamic in an acidic sulphate soil was evaluated under greenhouse conditions using a complete randomized design with two factors (site and horizon).
\end{abstract}

'Universidad de Córdoba, Departamento de Ingeniería Agronómica y Desarrollo Rural, Carrera 6 No. 76 -103, Montería - Colombia, Telefax (57-4) 786 0255. Email: ecabralesh@sinu.unicordoba.edu.co

${ }^{2}$ Universidad Nacional de Colombia, Sede Palmira 
Three different levels and four replicates were evaluated within each factor for a total of 36 experimental units. Total S, organic S, inorganic S, extractable $S$ and soluble sulphate variables were evaluated since the soil was fully saturated until it was air-dried. Humidity dynamics affected sulphate content only, which decreased with soils drainage.

Key words: acid sulphate soils, total sulfur, organic sulfur, inorganic sulfur, sulphates.

\section{INTRODUCCIÓN}

Los suelos sulfatados ácidos (SSA) son característicos de las zonas bajas que se inundan con frecuencia o en su efecto permanecen gran parte del tiempo inundados. También se encuentran en los deltas y estuarios de los ríos, en los que se generan ácidos solubles (ácido sulfúrico), producto de mezclas de sedimentos minerales y/u orgánicos ricos en hierro y azufre. Cuando se drenan estos suelos, los sulfuros se oxidan y se forman horizontes sulfúricos, con $\mathrm{pH}$ extremadamente ácidos $(<4)$; por otro lado, poseen altas concentraciones de azufre (>100 ppm) y aluminio intercambiable $\left(>5 \mathrm{cmol}(+) \mathrm{Kg}^{-1}\right)$ (Fitzpatrick, 1996; Dent y Dawson, 2000).

El origen de Ios SSA es variado; su origen puede ser marino, lacustre o halohidrotérmico. La formación data más allá de 10000 años, posiblemente en el Holoceno, cuyo mineral dominante es la Pirita ( $\mathrm{Fe}_{2} \mathrm{~S}$ ) (Pons y Breemen, 1982; Bello y Gómez, 2001). Para el año 2000 se reportaban que en el mundo existían aproximadamente 24 millones ha con problemas de SSA, de los cuales $16.6 \%$ se encuentra en América Latina (Dent y Dawson, 2000). En Colombia, se encuentran en los departamentos de Córdoba, Bolívar, Cesar, Valle del Cauca y Boyacá principalmente, pero no se conoce su totalidad. Córdoba posee aproximadamente 30000 ha, ubicadas en su gran mayoría en suelos del Valle del Sinú, cifra que se estima de carácter creciente por el drenado continuo de las ciénagas (Cabrales, 2006). Este incremento del área total y la aparición de nuevas áreas con SSA, hace plantear estudios en torno a ellos, ya que son quizás los suelos menos estudiados, si se comparan con otro tipo de suelos. Uno de los problemas que poseen estos suelos, es el desconocimiento de la dinámica del azufre, y en el caso particular, se desconoce la concentración de azufre de los SSA del Valle del Sinú, lo que ha conllevado al uso de fertilizantes ricos en azufre, con algunos resultados positivos a la aplicación. Con base en lo anterior, esta investigación tuvo como objetivo estudiar la dinámica del azufre en los tres primeros horizontes de un suelo sulfatado ácido del municipio de Ciénaga de Oro - Córdoba, en función al drenado del mismo, para lo cual se determinaron las concentraciones de S total, S orgánico, S inorgánico, S extraíble y sulfatos $\left(\mathrm{SO}_{4}\right)$ desde su completa saturación hasta que quedó seco al aire, bajo la hipótesis de que la dinámica del azufre depende del contenido de humedad del suelo y de la profundidad de muestreo.

\section{MATERIALES Y MÉTODOS}

La investigación se realizó en tres sitios o fincas (San Antonio, La Gran China y El Deseo) ubicadas en el transecto Berastegui - La Gran China - Mimbre, municipio de Ciénaga de Oro, departamento de Córdoba, orientado hacia la Ciénaga Grande de 
Lorica, con rumbo noreste. De estos sitios se colectaron muestras de los tres primeros horizontes y se transportaron al invernadero de la Universidad de Córdoba, seguidamente se sometieron a saturación por un tiempo de 10 meses. Para lo cual se utilizó un diseño completamente al azar con arreglo factorial $3 \times 3$ (tres sitios y tres horizontes) y cuatro repeticiones. Para el análisis se utilizó el paquete estadístico SAS y la comparación de medias se hizo por la DMS (Diferencia Mínima Significativa), para cada época de muestreo (contenido de humedad). De igual manera, se hizo análisis de regresión entre contenido de humedad y cada una de fracciones de azufre determinadas, con el objeto de encontrar los modelos matemáticos y sus respectivas tendencias para cada horizonte (Díaz, 2002). El muestreo de suelo para los análisis se inició cuando el suelo estaba completamente saturado, cada mes por un tiempo de siete meses, hasta que el suelo quedó seco al aire. A cada muestra se le determinaba el contenido de humedad y los parámetros que se señalan en la tabla 1 .

Tabla 1. Parámetros evaluados y sus respectivas metodologías utilizadas.

\begin{tabular}{lcl}
\hline \multicolumn{1}{c}{ Parámetro } & \multicolumn{1}{c}{ Metodología } & \multicolumn{1}{c}{ Fuente } \\
\hline S total & Digestión del nitrato de potasio/ácido nítrico & Beaton et al. (1997) \\
S orgánico & Bardsley y Lancaster & Beaton et al. (1997) \\
S inorgánico & Por diferencia S total - S orgánico & Beaton et al. (1997) \\
S extraíble & Extracción con fosfato de calcio monohidratado & ICA (1989) y Lisle et al. (1994) \\
Sulfato & Método turbidimétrico & ICA (1993) \\
\hline
\end{tabular}

\section{RESULTADOS Y DISCUSIÓN}

\section{Azufre total}

El contenido promedio de S total de los tres suelos estudiados fue de $21393.6 \mathrm{ppm}$. Entre los sitios se encontró diferencia altamente significativa $(\operatorname{Pr}<0.001)$ (Tabla 2) siendo el tercer sitio (El Deseo) el que mayor $S$ total tuvo (30711.7 ppm) y San Antonio el de menor concentración (10737.4 ppm) (Tabla $3)$. Esto indica que hay una tendencia creciente hacia la Ciénaga Grande de Lorica, posiblemente se deba a que son suelos con más limitaciones en el drenaje natural, por ende hay mayor capacidad y oportunidad de almacenamiento de elementos, entre ellos el azufre.

Las concentraciones obtenidas en esta investigación están en el rango reportado
(3000 - 35000 ppm de S total) en suelos cenagosos de los Estados Unidos (Whitehead, 1970 citado por Grandeth, 2003). De éste sólo se exceptúa el contenido encontrado en el primer horizonte del tercer sitio (El Deseo) con 56563.1 ppm, lo cual se debe posiblemente a que es una zona mucho más baja, por ende tuvo mayor posibilidad de acumular mayor cantidad de azufre, no sólo por el mal drenaje, sino que a él le llegan desde las partes más altas. Estos contenidos están muy por encima de las 2166 ppm que reporta Burbano (2001) en suelos de incidencia volcánica en los Altiplanos de Pastos e Ipiales (Colombia), aunque no son SSA, son muy ricos en azufre.

Las concentraciones encontradas ( $>7452.5$ ppm o $0.74 \%$ de $\mathrm{S}$ total en el tercer horizonte en San Antonio) están por encima del $0.7 \%$ 
Tabla 2. Cuadrados medios del ANAVA y nivel de significancia estadística para S total, orgánico, inorgánico y extraíble en función al drenado de un suelo sulfatado ácido.

\begin{tabular}{|c|c|c|c|c|c|c|c|c|}
\hline Factor & G.L. & 1 & 2 & 3 & 4 & 5 & 6 & 7 \\
\hline \multicolumn{9}{|c|}{ Cuadrados medios del ANAVA y nivel de significancia estadística para S total } \\
\hline Sitio & 2 & $1.1 \mathrm{E}+9^{* * *}$ & $1.1 \mathrm{E}+9^{* * *}$ & $1.2 \mathrm{E}+9 * * *$ & $1.2 \mathrm{E}+9^{* * *}$ & $1.2 \mathrm{E}+9^{* * *}$ & $1.2 \mathrm{E}+9^{* * *}$ & $1.3 \mathrm{E}+9 * * *$ \\
\hline Horizonte & 2 & $1.1 \mathrm{E}+9 * * *$ & $9.8 \mathrm{E}+8^{* * *}$ & $1.2 \mathrm{E}+9 * * *$ & $1.1 \mathrm{E}+9^{* * *}$ & $9.3 \mathrm{E}+8^{* * *}$ & $8.9 \mathrm{E}+8^{* * *}$ & $8.6 \mathrm{E}+8^{* * *}$ \\
\hline Sitio*Horiz & 4 & $5.4 \mathrm{E}+8^{* * *}$ & $5.0 \mathrm{E}+8^{* * *}$ & $5.5 \mathrm{E}+8^{* * *}$ & $5.7 \mathrm{E}+8^{* * *}$ & $5.9 \mathrm{E}+9^{* * *}$ & $5.6 \mathrm{E}+8^{* * *}$ & $5.5 \mathrm{E}+8^{* * *}$ \\
\hline Error & 27 & $8.5 E+6$ & $6.9 E+6$ & $1.1 \mathrm{E}+7$ & $6.4 \mathrm{E}+6$ & $6.2 E+6$ & $4.4 \mathrm{E}+6$ & $1.9 \mathrm{E}+7$ \\
\hline C.V. (\%) & & 13.98 & 12.39 & 15.43 & 11.72 & 11.44 & 10.10 & 20.31 \\
\hline \multicolumn{9}{|c|}{ Cuadrados medios del ANAVA y nivel de significancia estadística para S orgánico } \\
\hline Finca & 2 & $2.1 \mathrm{E}+7^{* * *}$ & $2.8 \mathrm{E}+7^{* * *}$ & $2.22^{* * *}$ & $2.5 \mathrm{E}+7^{* * *}$ & $2.8 \mathrm{E}+7^{* * *}$ & $3.25^{* * *}$ & $2.9 \mathrm{E}+7^{* * *}$ \\
\hline Horizonte & 2 & $1.1 \mathrm{E}+7^{* * *}$ & $1.3 \mathrm{E}+7^{* * *}$ & $1.60 * * *$ & $1.4 \mathrm{E}+7^{* * *}$ & $1.7 \mathrm{E}+7^{* * *}$ & $2.01 * * *$ & 1.7E+7*** \\
\hline Finca*Horiz & 4 & $6.8 \mathrm{E}+6^{* * *}$ & $8.0 \mathrm{E}+6^{* * *}$ & $0.77^{* * *}$ & $1.0 \mathrm{E}+7^{* * *}$ & $1.0 \mathrm{E}+7^{* * *}$ & $0.75^{* * *}$ & $1.3 \mathrm{E}+7^{* * *}$ \\
\hline Error & 27 & $7.1 \mathrm{E}+4$ & $3.2 \mathrm{E}+5$ & 0.005 & $9.2 \mathrm{E}+4$ & $4.2 \mathrm{E}+4$ & 0.02 & $2.2 \mathrm{E}+4$ \\
\hline C.V. (\%) & & 8.68 & 17.94 & 0.96 & 9.61 & 6.21 & 1.99 & 5.06 \\
\hline
\end{tabular}

Cuadrados medios del ANAVA y nivel de significancia estadística para $\mathbf{S}$ inorgánico

$\begin{array}{lllllllll}\text { Finca } & 2 & 1.4 \mathrm{E}+9 * * * & 1.3 \mathrm{E}+9^{* * *} & 1.4 \mathrm{E}+9^{* * *} & 1.4 \mathrm{E}+9 * * * & 1.5 \mathrm{E}+9^{* * *} & 1.5 \mathrm{E}+9^{* * *} & 1.5 \mathrm{E}+9^{* * *} \\ \text { Horizonte } & 2 & 1.0 \mathrm{E}+9 * * * & 9.0 \mathrm{E}+8^{* * *} & 1.1 \mathrm{E}+9^{* * *} & 1.1 \mathrm{E}+9^{* * *} & 8.9 \mathrm{E}+8^{* * *} & 8.6 \mathrm{E}+8^{* * *} & 8.1 \mathrm{E}+8^{* * *} \\ \text { Finca*Horiz } & 4 & 6.1 \mathrm{E}+8^{* * *} & 5.7 \mathrm{E}+8^{* * *} & 6.2 \mathrm{E}+8^{* * *} & 6.5 \mathrm{E}+8^{* * *} & 6.6 \mathrm{E}+9 * * * & 6.1 \mathrm{E}+8^{* * *} & 6.3 \mathrm{E}+8^{* * *} \\ \text { Error } & 27 & 8.9 \mathrm{E}+6 & 7.3 \mathrm{E}+6 & 1.1 \mathrm{E}+7 & 6.7 \mathrm{E}+6 & 6.3 \mathrm{E}+6 & 4.0 \mathrm{E}+6 & 1.9 \mathrm{E}+7 \\ \text { C.V. }(\%) & & 16.82 & 14.97 & 18.75 & 14.09 & 13.61 & 11.38 & 23.73\end{array}$

Cuadrados medios del ANAVA y nivel de significancia estadística para $\mathrm{S}$ extraíble

$\begin{array}{lllllllll}\text { Sitio } & 2 & 7.3 \mathrm{E}+6^{* * *} & 2.1 \mathrm{E}+7^{* * *} & 1.7 \mathrm{E}+7^{* * *} & 1.3 \mathrm{E}+7^{* * *} & 1.8 \mathrm{E}+7^{* * *} & 1.1 \mathrm{E}+7^{* * *} & 9.3 \mathrm{E}+6^{* * *} \\ \text { Horizonte } & 2 & 3.5 \mathrm{E}+6^{* * *} & 1.0 \mathrm{E}+7^{* * *} & 1.3 \mathrm{E}+7^{* * *} & 7.8 \mathrm{E}+6^{* * *} & 7.8 \mathrm{E}+6^{* * *} & 9.6 \mathrm{E}+6^{* * *} & 4.8 \mathrm{E}+6^{* * *} \\ \text { Sitio*Horiz } & 4 & 2.4 \mathrm{E}+6^{* * *} & 1.4 \mathrm{E}+7^{* * *} & 1.5 \mathrm{E}+7^{* * *} & 1.0 \mathrm{E}+7^{* * *} & 1.3 \mathrm{E}+7^{* * *} & 1.1 \mathrm{E}+7^{* * *} & 6.4 \mathrm{E}+6^{* * *} \\ \text { Error } & 27 & 1.0 \mathrm{E}+5 & 3.0 \mathrm{E}+5 & 7.9 \mathrm{E}+4 & 8.5 \mathrm{E}+4 & 1.0 \mathrm{E}+5 & 1.4 \mathrm{E}+5 & 1.5 \mathrm{E}+5 \\ \text { C.V. }(\%) & & 12.87 & 19.63 & 9.52 & 10.49 & 11.77 & 14.62 & 15.60\end{array}$

Cuadrados medios (CM) del ANAVA y nivel de significancia estadística para $\mathbf{S}$ sulfato

\begin{tabular}{lllllllll} 
Sitio & 2 & $0.07^{*}$ & $0.62^{* *}$ & $0.92^{*}$ & $1.96^{* * *}$ & $700.9^{*}$ & $985.8^{* *}$ & $0.20^{* * *}$ \\
Horizonte & 2 & $1.32^{* * *}$ & $1.99^{* * *}$ & $0.14 \mathrm{NS}$ & $1.38^{* * *}$ & $12103.8^{* * *}$ & $11324^{* * *}$ & $2.95^{* * *}$ \\
Sitio*Horiz & 4 & $0.71^{* * *}$ & $0.39^{* *}$ & $0.07 \mathrm{NS}$ & $0.64^{* * *}$ & $3961.7^{* * *}$ & $1598.9^{* * *}$ & $0.05^{* * *}$ \\
Error & 27 & 0.01 & 0.07 & 0.018 & 0.07 & 155.8 & 123.66 & 0.008 \\
C.V. (\%) & & 2.12 & 5.16 & 8.72 & 5.83 & 19.03 & 17.94 & 2.42 \\
\hline
\end{tabular}

* Significativo al 5\%; ${ }^{* *}$ significativo al 1\%; ${ }^{* *}$ significativo a menos del $1 \%$; NS no significativo.

que reporta Thomas y Varley (1982), para considerárseles como suelos sulfatados, por lo tanto, no hay duda que los suelos estudiados son SSA. Los altos contenidos de azufre total en estos suelos se deben a la riqueza en azufre que poseen los materiales parentales de la formación Ciénaga de Oro que le dieron origen, ya que son ricos en sulfuros de hierro y yeso entre otros.
Estadísticamente se encontró que el drenado en estos suelos no influyó en el contenido de azufre total; sin embargo, se observó una ligera variación en función al drenado, pero el coeficiente de determinación indicó que el drenado no explica satisfactoriamente los cambios de las concentraciones de S total. Esto indica, que el S total de los SSA requiere de muchos años para mostrar su dinámica. 
La variabilidad que se observa en la tabla 3 se debe a la heterogeneidad de estos suelos y al mismo complejo de materiales parentales que le dieron origen. El alto contenido de azufre que muestra el primer horizonte se debe a que a éste se le suma el azufre que le aporta la materia orgánica, mientras que el aporte que reciben los horizontes inferiores, es mucho menor. El hecho de que el contenido de azufre total en estos suelos se mantenga en función al drenado, indica que con esta actividad, difícilmente se disminuye su contenido en estos suelos, es decir, que su rehabilitación suele ser muy costosa y a largo plazo, por lo que resulta más económico dejarlos como reserva natural. Esta apreciación concuerda con la de Brinkman (1982) en cuanto a que los SSA por su lenta evolución deben dejarse como estuario para preservación de la flora y fauna.

El análisis de los contenidos en cada horizonte, señala que el $\mathrm{S}$ total disminuye con la profundidad (Tabla 3), lo cual se debe posiblemente al aporte de azufre que hace la materia orgánica, que desde luego fue mayor en el primer horizonte y decrece con la profundidad. Esta tendencia concuerda con los resultados de Thomas y Varley (1982), pero contradice lo encontrado por Dend y Raiswell (1982) en suelos del Valle del Río Gambia (Senegal), que el contenido de azufre total aumentaba con la profundidad. De esta tendencia se exceptúa el segundo, que posee un horizonte orgánico (Obe) sepultado que sesga dicha tendencia.

\section{Azufre orgánico}

Los SSA del Valle del Sinú poseen un alto contenido de $\mathrm{MO}(>10 \%$, determinada por combustión seca); a pesar de ello, los promedios de S orgánico de los sitios estudiados no alcanzaron a superar las concentraciones de S inorgánico (Tabla 3), y en ninguno de los sitios estudiados, lo cual indica que posiblemente el origen del azufre de los SSA del Valle del Sinú es inorgánico (materiales ricos en azufre de la formación Ciénaga de Oro, como la pirita, yeso, etc) más que orgánico. Todas las concentraciones de S orgánico encontrados

Tabla 3. Concentraciones de azufre total, orgánico, inorgánico y extraíble en los tres primeros horizontes de tres suelos sulfatados ácidos del Valle del Sinú - Colombia.

\begin{tabular}{ccccccc}
\hline \multirow{2}{*}{ Horizontes } & S total & \multicolumn{2}{c}{ S Organico } & \multicolumn{2}{c}{ S inorganico } & S extraible \\
& ppm & ppm & \% & ppm & \% & ppm \\
\hline Ap $(0-9 \mathrm{~cm})$ & 13733.3 & 4481.3 & 32.6 & 9252.0 & 67.4 & 1266.6 \\
Bw1 $(9-32 \mathrm{~cm})$ & 11026.5 & 3768.4 & 34.2 & 7258.1 & 65.8 & 1079.0 \\
Bw2 $(32-43 \mathrm{~cm})$ & 7452.5 & 2522.4 & 33.8 & 4930.0 & 66.2 & 1173.8 \\
& & Finca La Gran China & & & \\
Ap $(0-20 \mathrm{~cm})$ & 25915.9 & 4687.0 & 18.1 & 21229.0 & 81.9 & 1729.1 \\
Obe $(20-26 \mathrm{~cm})$ & 17685.8 & 7091.9 & 40.1 & 10593.9 & 59.9 & 3295.7 \\
Bwg $(26-95 \mathrm{~cm})$ & 24593.3 & 1498.2 & 6.1 & 23095.1 & 93.9 & 796.6 \\
& & Finca El Deseo & & \\
Ap $(0-5 \mathrm{~cm})$ & 56563.1 & 1534.4 & 2.7 & 55028.7 & 97.3 & 1213.2 \\
Bw1g $(5-38 \mathrm{~cm})$ & 19287.3 & 1458.6 & 7.6 & 17828.7 & 92.4 & 763.9 \\
Bw2g $(38-60 \mathrm{~cm})$ & 16284.6 & 1645.3 & 10.1 & 14639.3 & 89.9 & 780.8 \\
\hline
\end{tabular}


en los tres horizontes de los sitios estudiados, están por encima de las concentraciones (1246 ppm) que reporta Burbano (2001) en el departamento de Nariño (Colombia), Io cual obedece posiblemente a que estos suelos no son sulfatados, por ende tendrán menor concentración de S orgánico. Sin embargo, los porcentajes encontrados están en los rangos (15 - 63\%) que reporta este mismo autor en estudios de suelos en distintas zonas de Colombia.

Estadísticamente se encontraron diferencias altamente significativas $(P r<0.001)$ entre sitios, de igual manera entre horizontes y la interacción sitio $\mathrm{x}$ horizonte (Tabla 2); por lo que se puede afirmar, que los suelos sulfatados del Valle del Sinú son muy heterogéneos y que la concentración de $\mathrm{S}$ orgánico dependerá de la ubicación geográfica y profundidad de muestreo. El mayor contenido (4425.7 ppm) se encontró en el segundo sitio, lo cual se debe al horizonte orgánico sepultado que posee, que incrementa el promedio de S orgánico en este sitio, no sólo en este horizonte, sino a los dos adyacentes. En términos generales, se nota una tendencia decreciente del contenido de S orgánico hacia la Ciénaga Grande de Lorica, lo cual se debe posiblemente al sistema de labranza a que son sometidos estos suelos, y que difieren de un sitio a otro, toda vez que la aireación (inducida o natural) de los suelos influye en la mineralización del azufre orgánico.

Estadísticamente se encontró que el drenaje de estos suelos no influyó en el contenido de S orgánico; sin embargo, en los horizontes segundo de la finca La Gran China, y primero y segundo de El Deseo, se observó una ligera tendencia. Hecho el análisis de regresión, se determinó que el drenado de los suelos no explica satisfactoriamente esta variabilidad, es decir, que existen otras variables de mayor peso que influyen en la variabilidad del $\mathrm{S}$ orgánico de estos suelos, como por ejemplo $\mathrm{pH}$ que regula no solo población microbial, sino su diversidad y actividad, lo cual será materia de estudio posteriormente. La pobre influencia que tuvo el drenaje en la dinámica del S orgánico, se debe a la deficiencia de oxígeno que caracterizan a estos, producto de su microporosidad, lo cual reduce la mineralización del azufre por los microorganismos, concordando esto con las afirmaciones de Blasco (1970), quien plantea que suelos pobres en oxígeno se caracterizan por su baja mineralización. A estas condiciones físicas adversas se le suman las químicas, especialmente el pH extremadamente ácido, que limita la actividad microbiana del suelo.

\section{Azufre inorgánico}

Se encontraron diferencias altamente significativas $(\operatorname{Pr}<0.0001)$ entre sitios, entre horizontes e interacción sitio $\mathrm{x}$ horizonte (Tabla 2), siendo el tercer sitio (El Deseo) el de mayor concentración (29165.6 ppm) y la de menor fue el primer sitio (San Antonio) (7134.8 ppm) (Tabla 3), en la cual se nota una tendencia creciente hacia la Ciénaga Grande de Lorica, lo cual se debe posiblemente a la disminución del contenido de materia orgánica; es decir, la cantidad de $S$ inorgánico es inversamente proporcional al contenido de materia orgánica. Estos contenidos de S inorgánico están muy por encima de la concentración de 1921 ppm reportada por Burbano (2001) en los suelos de Nariño, lo cual se debe a la misma riqueza de azufre que caracterizan a los SSA del Valle del Sinú, a ello se le suma la baja capacidad de drenaje que poseen, ubicación en las zonas más bajas, microporosidad, etc.

Los análisis de las concentraciones de S inorgánico encontrados en los horizontes en conjunto de los tres sitios, mostró diferencias 
altamente significativas $(P r<0.001)$ entre horizontes, siendo el primer horizonte el que exhibe la mayor concentración de S inorgánico (28503.2 ppm), mientras que la menor concentración (11893.6 ppm), se encontró en el segundo horizonte. Esta diferencia se debe posiblemente a que el segundo horizonte mostró un mayor contenido de materia orgánica, lo que ha podido influir en aportar menor cantidad de esta fracción. En términos generales se nota que la cantidad de $S$ inorgánico disminuye con la profundidad, solo se exceptúa el segundo sitio (La Gran China) por poseer un horizonte orgánico sepultado que sesga dicha tendencia. Estadísticamente se encontró que el drenado no influyó en la concentración de S inorgánico, lo que indica que la mineralización y dinámica del S inorgánico no depende únicamente del drenado o aireación del suelo, sino de otros factores que serán materia de estudio posteriormente. Los altos contenidos de $S$ inorgánico que se encontraron (>4900 ppm) reflejan la riqueza de minerales ricos en azufre, como pirita, yeso, y jaroisita, entre otros.

\section{Azufre extraible}

Las concentraciones de $\mathrm{S}$ extraíble encontrados en los sitios estudiados, mostraron diferencias altamente significativas $(\operatorname{Pr}<0.001)$ entre sitios, entre horizontes e interacción sitios $x$ horizonte (Tabla 2); siendo el segundo sitio (La Gran China) el que arrojó la mayor concentración de $S$ extraíble, con 1940.5 ppm y la que menos concentración mostró fue el tercer sitio (El Deseo) con 919.3 ppm (Tabla 3), esta diferencia se debe posiblemente a la actividad microbial que tiene cada sitio en particular. El menor contenido que muestra el primer sitio se debe posiblemente a que este sitio permanece mayor tiempo bajo inundación (suelo saturado) y la actividad biológica por ende será menor que los otros dos sitios que permanecen menos tiempo saturado. Lo anterior coincide con las afirmaciones de Coyne (2000) y Correa y Padilla (2001), quienes encontraron que bajo condiciones de saturación se ve afectada la actividad biológica del suelo: mucha humedad reduce el oxígeno del suelo, por lo tanto se disminuye la oxidación del azufre en estos suelos.

Al observar la variación en conjunto que hubo entre los horizontes de los tres sitios en estudio, se notó una diferencia altamente significativa $(\operatorname{Pr}<0.001)$, siendo el primer horizonte el que mayor concentración de $S$ extraíble mostró con 1402.9 ppm, y el de menor concentración fue el tercer horizonte, con 917.1 ppm en promedio (Tabla 3). Lo anterior indica que el contenido de $\mathrm{S}$ extraíble decrece con la profundidad en el suelo, lo cual se debe a que en la medida que se profundiza, la actividad microbial decrece, al igual que el contenido de azufre orgánico e inorgánico, por ende la concentración de S extraíble u oxidable también decrece. Las concentraciones de S extraíble de estos suelos está muy por encima del promedio (10 - 20 ppm), de los suelos agrícolas que reporta Edmeades (1994). De igual manera, las concentraciones del primer horizonte están por encima de las 900 ppm que reporta Correa y Padilla (2001) en el mismo horizonte en suelos SSA del Valle del Sinú, explicando esto la diversidad de suelos sulfatados que se encuentran a lo largo y ancho en el Valle del Sinú. Esto es, que el contenido de $\mathrm{S}$ extraíble depende de sitio de muestreo; sin embargo, en el transecto no se encontró una tendencia espacial, lo cual se debe posiblemente a la misma dinámica de cada suelo en particular, producto del manejo agrícola que año tras año recibe, algunos con mayor intensidad que otros. Estadísticamente se encontró que el drenado no explica significativamente $(P r<0.05)$ la concentración de S extraíble y su alto contenido se debe posiblemente a 
que las diferentes formas de azufre orgánico y mineral pueden ser extraídas por el método de fosfato monocálcico en cantidades similares sin tener dependencia de la humedad. De estos, se exceptúa el segundo horizonte de la finca San Antonio que muestra una diferencia significativa $(\operatorname{Pr}<0.05)$, indicando que el drenado disminuye la cantidad de S extraíble del suelo SSA; sin embargo, el bajo coeficiente de determinación $(16,4 \%)$ indica que el drenado explica esta variabilidad en muy bajo porcentaje y el resto lo explican otras variables. Esto contradice lo reportado por Coyne (2000) quien afirma que la oxidación del azufre es directamente proporcional a la aireación del suelo, mientras que en esta investigación se encontró que cuando se drena, si bien hay una ligera tendencia, esta no es significativa. Posiblemente se deba a que los suelos sulfatados estudiados, permanece mucho tiempo saturados y muchas veces inundados, por otro lado, al ser drenados, su acidificación aumenta, lo que a su vez podría estar influyendo negativamente en la oxidación del azufre.

\section{Sulfato soluble $\left(\mathrm{SO}_{4}{ }^{=}\right)$}

Las concentraciones de sulfato variaron entre sitios, horizontes, interacción (Tabla 3 y Figuras 1,2 y 3) de igual manera en la medida que el suelo fue drenado. La mayor concentración (338.8 ppm) se encontró en La Gran China con el suelo completamente saturado; sin embargo, entre éste sitio y el primero (San Antonio) no hubo diferencia estadística, pero entre estos dos y el tercero (El Deseo), se observó diferencia altamente significativa $(\operatorname{Pr}<0.0001)$ como se observa en la Tabla 2. En términos generales, esta variable no mostró una clara tendencia en función a la lejanía o cercanía de la ciénaga Grande de Lorica, debido posiblemente a la heterogeneidad de los suelos y a la misma dinámica de manejo a que están sometidos cada uno de ellos, producto del sistema y tiempo de explotación que poseen.

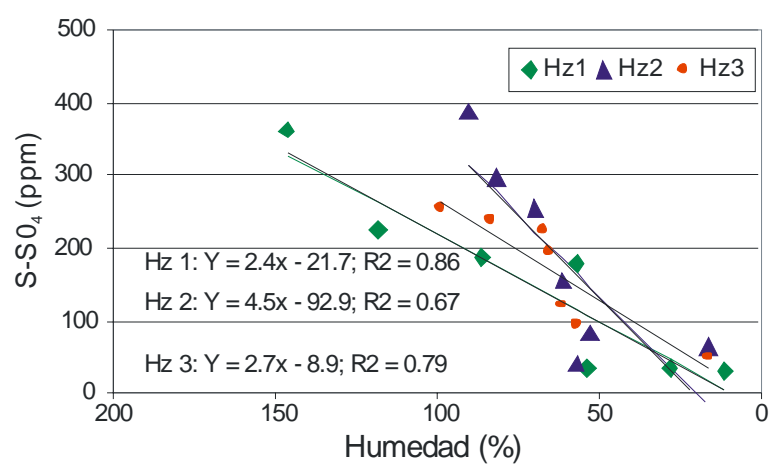

Figura 1. Variación del sulfato en función al drenado del suelo en los tres primeros horizontes de la finca San Antonio, Valle del Sinú - Colombia.

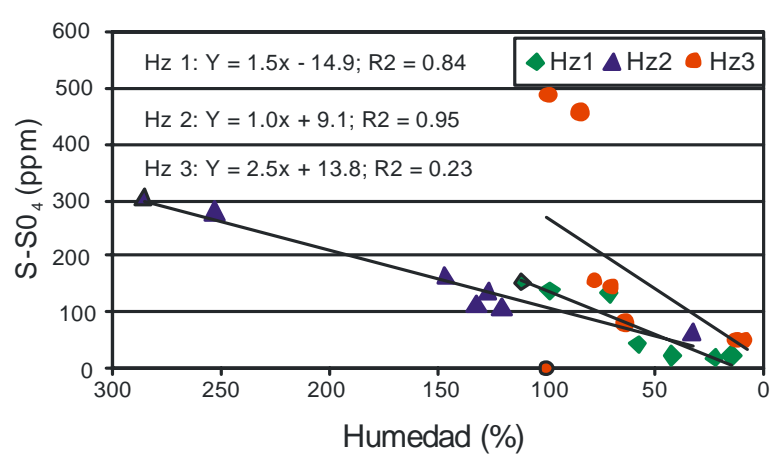

Figura 2. Variación del sulfato en función al drenado del suelo en los tres primeros horizontes de la finca La Gran China, Valle del Sinú - Colombia.

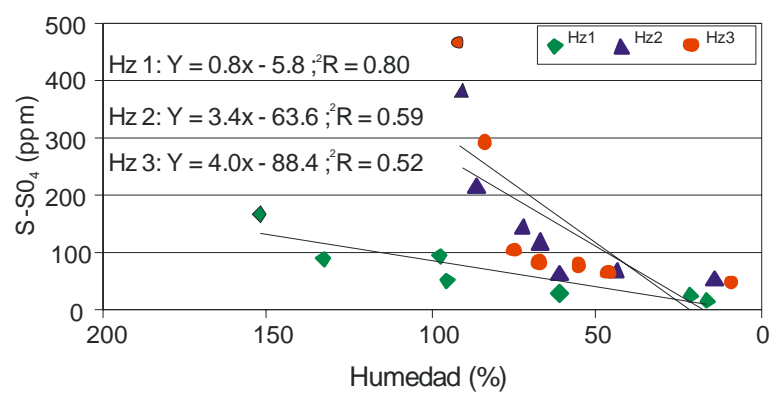

Figura 3. Variación del sulfato en función al drenado del suelo en los tres primeros horizontes de la finca El Deseo, Valle del Sinú - Colombia.

Los altos contenidos de sulfato soluble en las etapas iniciales de la investigación (suelo completamente saturado), se deben posiblemente al largo tiempo (10 meses) que 
duró el suelo saturado, lo cual es posible que haya contribuido en la solubilización parcial del sulfato. Sin embargo, en la medida que se drenó el suelo, se observó que las concentraciones de sulfatos decrecieron, desde más de 300 ppm hasta llegar a concentraciones de 18.4 ppm en el primer horizonte del tercer sitio, cuando el suelo se encontraba en el rango seco (seco al aire). Esta tendencia decreciente en función al drenado del suelo, se debe posiblemente a que parte del sulfato se perdió en el lixiviado (drenaje) y/o se hayan formado compuestos complejos e insolubles. Es de anotar que en la investigación, la mayor parte del agua se perdió a través del drenaje y no por evapotranspiración. En todos los casos, las concentraciones de sulfato encontradas en estos suelos, están por encima de los reportados en trabajos similares como los de Correa y Padilla (2001), quienes encontraron en SSA del Valle del Sinú concentraciones de sulfatos que oscilaron entre 2.51 y 14.7 ppm, esta diferencia se debe posiblemente a que estos suelos poseen mayor cantidad de azufre que los estudiados por estos autores.

Estadísticamente se encontró diferencia altamente significativa entre sitios, horizontes e interacción sitio $x$ horizonte, siendo el segundo y tercer horizonte los que mostraron la mayor concentración, lo cual se debe posiblemente a la lixiviación de este ion en el agua de drenaje en los siete meses en que estuvo sometido, lo cual coincide con lo encontrado por Herrera y Baquero (1993) en suelos del Valle del Sinú. El coeficiente de determinación que se muestra en las figura 1, 2 y 3, señala que el drenado influye en la dinámica del sulfato en los suelos SSA del Valle del Sinú; sin embargo, hay otros factores que también influyen en esta variable.

Esta tendencia decreciente con el drenado del suelo, se debe posiblemente a que el sulfato habría reaccionado con las bases del suelo, principalmente con el calcio y precipitaría bajo las formas de compuestos complejos e insolubles tales como el yeso $\left(\mathrm{CaSO}_{4} 2 \mathrm{H}_{2} \mathrm{O}\right)$, lo anterior coincide con las afirmaciones de Grandeth (2003), quien afirma que en los suelos del Valle del Sinú hay posibilidades que se formen suelos yesíferos cuando estos son drenados, ya que son suelos con altas concentraciones de azufre y calcio. También es factible que el hierro bajo condiciones de deficiencias de oxígeno, haya reaccionado con el azufre y hubiesen formado compuestos como la pirita y otros compuestos de azufre distintos a los sulfatos, lo cual concuerda con las afirmaciones de Lindsay (1979), quien plantea que la actividad del hierro y del azufre dependen de las condiciones redox del medio. Por otro lado, la producción de sulfatos, además de la microflora del suelo, está influenciada por otros factores como $\mathrm{pH}$, oxígeno en el suelo y en menor grado la temperatura, resultados que concuerdas con los reportados por Coyne (2000). Es de anotar, que los resultados de esta investigación también concuerdan con los reportados de Kittams y Attoe (1966) citado por Grandeth (2003), quienes no encontraron una consistente relación entre el $\mathrm{pH}$ y humedad con la oxidación del azufre. Por otro lado, esta tendencia decreciente con el drenado del suelo, también ha podido deberse a la disminución del $\mathrm{pH}$, toda vez que al disminuir el $\mathrm{pH}$ se incrementa la capacidad de intercambio aniónico y por ende la capacidad fijadora de aniones, como lo afirman Tisdale y Nelson (1991), en el sentido que las concentraciones de aniones solubles disminuyen en la medida que se disminuye el $\mathrm{pH}$ de los suelos, por el incremento de la capacidad de intercambio aniónico de los mismos. Estos resultados también coinciden con los encontrados por Safo y Oppong (1994), quienes encontraron que al disminuir el $\mathrm{pH}$ de 4.6 a 4.5 , las concentraciones de sulfatos bajaron de 4.8 a 4.6 ppm. 


\section{CONCLUSIONES Y RECOMENDACIONES}

- La humedad del suelo no influyó en los contenidos de azufre total, orgánico, inorgánico y extraíble, por lo tanto su determinación se puede hacer con cualquier contenido de humedad.

- En el área de estudio, el contenido de azufre orgánico decrece con la cercanía a la ciénaga Grande de Lorica, mientras que el inorgánico y el total lo hace en forma creciente.
- Se recomienda realizar trabajos similares al anterior, pero en otras zonas de suelos sulfatados del Valle del Sinú, para corroborar las tendencias encontradas al igual que realizar una caracterización de la microbiología de los suelos sulfatados y su dinámica.

- Debido a las condiciones físicas de estos suelos, principalmente su drenaje deficiente por su tipo de capilaridad, su habilitación suele ser muy costosa, por lo que resulta mejor no sólo económica, sino ambientalmente, dejarlos como área de reserva natural.

\section{BIBLIOGRAFÍA}

Beaton, J.; Burns, G. y Platou, J. 1997. Determination of sulphur in soils and plant material. Technical Bulletin Number 14, The Sulphur Institute, Washington D.C., $43 p$

Bello, J. y Gómez, M. 2001. Recuperación de suelos sulfatados ácidos en áreas del distrito de riego del Alto Chicamocha, Tibasosa (Boyacá). Tesis Ingeniero Agrónomo, Universidad Pedagógica y Tecnológica de Colombia, Tunja

Blasco, M. 1970. Curso de Microbiología de Suelos. IICA, San José, p178-207

Brinkman, R. 1982. Social and economic aspects of the reclamation of acid sulfate soil areas. Acid Sulfate Weathering, Wisconsin, p21-30

Burbano, H. 2001. El azufre en el suelo. Elementos secundarios (Ca, Mg y S) y silicio en la agricultura. SCCS, Santafé de Bogotá, p24-49
Cabrales, E. 2006. Microbiología de los suelos sulfatados ácidos del Valle del Sinú - Córdoba. Encuentro de experiencias en inventarios $y$ monitoreo biológico. Villa de Leyva

Cabrales, E.; Grandett, G. y Garcia, A. 2002. Caracterización de los suelos sulfatados ácidos del Valle del Sinú. IX Congreso de la Ciencia del Suelo, Cali, 3p

Correa, N. y Padilla, M. 2001. Estudio exploratorio de la macrofauna edáfica en suelos sulfatados ácidos, bajo diferentes manejos en la finca Soama de la planicie fluvio-lacustre del Valle del Sinú en el departamento de Córdoba. Tesis Especialista, Universidad de Cordoba, Montería

Coyne, M. 2000. Microbiología del suelo: un enfoque exploratorio. Paraninfo, Madrid, p12-60 
Dend, D. y Raiswell, R. 1982. Quantitative models to predict the rate and severity of acid sulphate development: a case study in the Gambia. Proceeding of the Bangkok Symposium on Acid sulphate Soils ISSC, Thailand, p73-85

Dend, D. y Dawson, B. 2000. The acid test. URL: http://www.staffi.Iboro.ac.uk. htm [Accedido: 25-03-2000]

Diaz, L. 2002. Estadística multivariada: inferencias y método. Universidad Nacional de Colombia, Santafé de Bogotá, p144-160

Edmeades, D. 1994. Some recent developments in sulphur research in New Zealand agriculture. Sulphur in Agriculture 18:3-8

Fitzpatric, R. 1996. Acid sulfate soil assessment. URL: http://ews.spirit [Accedido: 15-03-2003]

Grandeth, G. 2003. Evaluación general del estado actual y uso de los suelos en la parte baja de la planicie fluviolacustre del Medio Sinú en la zona de la Pozona, La Coroza y La Ceibita. CIUC, Universidad de Córdoba, Montería, p7-30

Herrera, J. y Baquero, J. 1993. Evaluación de la concentración de sulfatos en los suelos a diferentes contenidos de humedad. Seminario Investigativo Universidad de Córdoba, Facultad Ciencias Agrícolas, Montería, p4-37
ICA (Instituto Colombiano Agropecuario). 1989. El análisis de suelos, plantas y aguas para riego. Manual de Asistencia Técnica No. 47, Bogotá, ICA, p85-90

ICA. 1993 Manual de Análisis de Suelos, Plantas y Aguas para Riego, Bogotá, ICA, 35p.

Lisle, L.; Lefroy, R.; Anderson, G. y Blair, G. 1994. Methods for the measurement of sulphur in plants and soil. Sulphur in Agriculture 18:45-54

Lindsay, W. 1979. Chemical Equilibria in soils. Jhon Wiley y Sons, New York, p128-298

Pons, L. y Bremen, N. 1982. Factors influencing the formation of potential acidity in tidal swamps. Proceeding of the Bangkok Symposium on Acid Sulphate Soils, ISSC, Thailand, p37-49

Safo, E. y Oppong, S. 1994. The effects of legumes on soluble sulphate patterns in a Ghanaian forest soil: Studies with the winged beand, jakbean an cowpea. Sulphur in Agriculture 18:20-25

Thomas, P. y Varley, J. 1982. Soil survey of tidal sulphidic soils in the tropics: a case study. Proceeding of the Bangkok Symposium on Acid Sulphate Soils ISSC, Thailand, p52-53

Tisdale, S. y Nelson, W. 1991. Fertilidad de los suelos y fertilizantes. UTEHA, Ciudad de México, p310-334 